\title{
Protée
}

\section{La volonté de voir}

\section{Éthique et perception morale du sens}

\section{Sandra Laugier}

Volume 36, numéro 2, automne 2008

Éthique et sémiotique du sujet

URI : https://id.erudit.org/iderudit/019024ar

DOI : https://doi.org/10.7202/019024ar

Aller au sommaire du numéro

\section{Éditeur(s)}

Département des arts et lettres - Université du Québec à Chicoutimi

ISSN

0300-3523 (imprimé)

1708-2307 (numérique)

Découvrir la revue

\section{Citer cet article}

Laugier, S. (2008). La volonté de voir : éthique et perception morale du sens. Protée, 36(2), 89-100. https://doi.org/10.7202/019024ar

\section{Résumé de l'article}

Le présent article propose une approche pragmatique de l'éthique inspirée de la seconde philosophie de Wittgenstein. Après avoir présenté le projet d'une éthique " sans ontologie ", déplacée des concepts généraux vers l'exploration des pratiques et la vie ordinaire, elle met en évidence la nécessité d’une compétence éthique propre, articulée sur la capacité à voir le sens de l'action et sur la perception de l'importance. C'est à donner un contenu à ce concept de l'importance qu'on peut alors s'attacher, pour définir le sens moral comme base de la perception morale. d'utilisation que vous pouvez consulter en ligne.

https://apropos.erudit.org/fr/usagers/politique-dutilisation/ 


\section{LA VO LONTÉ DE VO IR ÉTHIQUEET PERCEPTION MORALE DU SENS}

SANDRA LAUGIER

Nous souhaitons proposer ici une approche de l'éthique inspirée de la seconde philosophie de Wittgenstein. Cela nous conduit à présenter, dans un premier temps, le projet d'une éthique «sans ontologie», déplacée vers l'exploration des pratiques et l'immanence de la vie ordinaire. Nous montrerons ensuite que cette exploration ne suffit pas en tant que telle et s'accomplit par la mise en ouvre, voire la formation, d'une compétence éthique: la capacité à saisir le sens de l'action et de la situation, la perception de ce qui est important. Enfin, nous mettrons en évidence une difficulté essentielle de cette perception morale, définie par le risque toujours ouvert de "passer à côté», par inattention ou négligence.

Dans ses Recherches philosophiques, Wittgenstein se demande quelle est l'importance de son travail, puisque sa recherche semble "détruire tout ce qui est grand et intéressant» (2004: §118). Mais qu'est-ce qui est important? Le but de la philosophie, pour Wittgenstein, est bien de redéfinir et déplacer notre idée de l'importance, de ce qui compte: ce que nous croyons important n'est que de l'air (Luftgebäude). Ce qui est réellement important nous est caché, non parce que ce serait dissimulé, privé ou introuvable, mais parce que c'est là, précisément, sous nos yeux. Il nous faut apprendre à voir, à être attentifs ou attentionnés. C'est cette volonté de voir qui définit la perception morale, mais peut-être aussi, comme nous essaierons de le montrer, la dimension ou la texture morale de toute expérience. La capacité à percevoir le détail de la vie ordinaire - saisir "ce qui compte» sur l'arrière-plan de la forme de vie - est un élément central de la compétence morale. C'est cette définition de la perception morale qui va nous servir de fil conducteur pour examiner une conception de l'éthique comme exploration perceptive, et donc comme attention, inséparablement sensible et conceptuelle, aux détails et expressions morales, afin de mettre en évidence la dimension aventureuse (pour reprendre une expression de Henry James) de toute exploration éthique.

\section{UNE ÉTHIQUE SANS MYTHOLOGIE ET SANS ONTOLOGIE}

Partons d'une redéfinition de la morale que propose, à partir de Wittgenstein, Cora Diamond. Il n'y a pas, pour elle, d'objet ou de sujet spécifique de la morale. 
Je vais différencier, pour commencer, deux approches de l'éthique. Nous pouvons penser qu'il y a une pensée et un discours ayant pour sujet ce en quoi consiste la vie bonne pour les êtres humains, ou quels principes d'action nous devons accepter, et que l'éthique philosophique sera alors la philosophie de ce domaine de pensée et de discours. Mais vous n'êtes pas obligé de penser cela: et Wittgenstein rejette cette conception de l'éthique. Tout comme la logique n'est pas, pour Wittgenstein, un sujet particulier, avec son propre corps de vérités, mais pénètre toute pensée, l'éthique n'a pas de sujet particulier; plutôt, un esprit éthique, une attitude envers le monde et la vie, peut pénétrer n'importe quelle pensée ou discours. (2000: 153) ${ }^{1}$

Cette éthique sans sujet particulier est alors une éthique de la perception, de ce à quoi ressemble (looks like) notre vie morale. "J'ai essayé, ajoute Diamond, de décrire certains traits de ce à quoi ressemble la vie morale, sans rien dire du tout de ce à quoi elle doit ressembler" (ibid.). Ce qui ne veut pas dire simplement décrire nos pratiques, ce que nous faisons.

La communication en matière de morale, comme en beaucoup d'autres, inclut l'exploration de ce qui permettra aux protagonistes de se rejoindre mutuellement: cela n'est pas «donné» par l'existence d'une "pratique». Nos pratiques sont exploratoires, et c'est en vérité seulement au travers d'une telle exploration que nous en venons à une vision complète de ce que nous pensions nous-mêmes, ou de ce que nous voulions dire.

(2004: 39)

C'est là une approche que Diamond tire d'une certaine lecture de Wittgenstein. L'éthique ne peut être décrite en simple référence à nos usages: notre idée de la morale (notre idéal) contribue aussi à forger nos pratiques morales, et l'usage ne suffit pas. Nos pratiques ne peuvent être fondatrices, parce que nous ne les connaissons pas. Cela rejoint ce que dit Diamond de nos pratiques comme exploratoires et non données: comme devant nous donner une vision complète de ce que nous pensons, disons ou voulons dire. Il s'agit d'explorer plus que d'argumenter, de «changer la façon dont nous voyons les choses» (ibid.). Cela conduit à transformer notre concept de la justification: la justification se produit au sein d'une forme de vie commune. D'où l'intérêt de Diamond pour l'examen de la littérature, des exemples, des énigmes, des petites histoires en éthique: l'usage de la littérature n'est pas seulement illustratif, mais, comme tout exemple, il nous fait voir plus clairement quelque chose de l'éthique et, plus précisément, de ce que nous attendons de l'éthique, de ce que nous voulons dire par elle.

Hilary Putnam s'est récemment inscrit, comme Diamond, dans l'héritage d'Iris Murdoch pour revendiquer une telle approche, qui consiste à faire attention à ce que nous disons, aux manières qu'ont nos expressions communes de nous guider ou de nous décevoir - ce qui n'est certainement pas la même chose que de renvoyer à "nos pratiques" ou conventions, car, comme le relève Diamond, il s'agit tout aussi bien, voire plus, de nos désaccords et de nos incompréhensions. Putnam note ainsi:

Il faut rompre entièrement avec une image étroite de ce qu'est un problème éthique. En mathématiques, on peut au moins dire que pour Wittgenstein les mathématiques ne sont pas une description. Mais on ne peut pas même dire cela de l'éthique, parce qu'il y a des propositions éthiques qui, tout en étant plus que des descriptions, sont aussi des descriptions. J'ai employé les mots de "fait enchevêtré» et là on est "enchevêtré» par des mots descriptifs comme "cruel», «impertinent", «inconsidéré». Des termes enchevêtrés, à la fois évaluatifs et descriptifs, sont au cour de notre vraie vie éthique. ${ }^{2}$

Putnam, comme Diamond, insiste sur le fait qu'il ne s'agit pas ici d'une approche "anti-théorique», mais de la diversité des voies qu'on peut prendre pour la description éthique (qui fait de l'éthique un bazar, un "motley", comme les mathématiques - et même un «motley» au carré [ «a motley squared», un super bazar]). Cela ne veut pas dire qu'on ne peut argumenter en éthique. Simplement, la réalité est bien plus «enchevêtrée» et demande un travail plus approfondi de l'imagination et de la perception, une éducation du sensible.

S'il y a un sens à vouloir revenir à "nos pratiques", c'est en examinant cet ensemble hétéroclite de 
pratiques de langage, ce que nous disons au cœur de l'ensemble grouillant de nos formes de vie, notre forme de vie dans le langage. Être réaliste consiste alors à revenir au langage ordinaire, à examiner nos mots et à leur prêter attention, à se soucier d'eux (de notre responsabilité envers nos mots et expressions; de la façon pour nous de ne pas les «laisser tomber»). Diamond critique une fascination en éthique qui serait comparable à celle qu'ont Frege et Russell, dans le champ de la logique, pour un idéal abstrait et mythologique: l'idéal d'une rationalité éthique «qui sous-tend les arguments moraux». Bien différemment, tout ne passe pas par des arguments en éthique.

Tout comme on peut faire des mathématiques en prouvant, mais aussi en traçant quelque chose et en disant, "regardez ceci», la pensée éthique procède par arguments et aussi autrement par des histoires et des images. L'idée que nous n'avons pas de pensée à moins que nous ne puissions réécrire notre point de vue sous la forme d'une argumentation d'une forme reconnue est l'effet d'une mythologie de ce qui est accompli par les arguments. (Diamond, 2004: introduction)

Nous imaginons comme Frege, qu'«il serait impossible à la géométrie de poser des lois précises si elle essayait de tenir des fils comme des lignes et des nœuds sur les fils comme des points" (cité par Diamond, 2004: 39). De façon parallèle, nous imaginons que la morale ne pourrait être pensée sans norme et sans nécessité, uniquement à partir de la réalité ordinaire et de ses nœuds et fils, de la tapisserie de la vie telle que Wittgenstein l'évoque à plusieurs reprises: «Un motif [déterminé] dans la tapisserie de la vie» (Lebensteppich) (1994: II, §862)

"Pas de fils ou de nœuds en logique ou en éthique!» Nous avons une idée erronée de la façon dont nos vies se rapportent à la rigueur de la logique, à l'obligation de l'éthique, à la nécessité des mathématiques. Nous sommes éblouis par les idéaux, et nous échouons à comprendre leur rôle dans notre langage. Quand nous sommes ainsi éblouis, nous sommes "en désaccord" avec nous-mêmes, notre langage, nos vies faites de fils et de nouds. La philosophie peut nous reconduire à «l'accord avec nous-mêmes", là où nous pensions le moins le trouver. La solution à l'énigme était juste là dans les nœuds et dans les fils. (Ibid.)
C'est ce qui caractérise «l'esprit réaliste»: comprendre que ce qui compte, ce qu'il faut regarder, ce sont les nœuds et les fils, le tissage de nos vies ordinaires. On retrouve ici une image commune à Henry James et à Wittgenstein, celle de l'image dans le tapis, qui évoque le tissage du conceptuel et de l'empirique.

Si la vie était une tapisserie, tel ou tel motif ne serait pas toujours complet et varierait de multiples façons. Mais nous, dans notre monde conceptuel, nous voyons toujours la même chose se répéter avec des variations. C'est ainsi que nos concepts saisissent. (Ibid. : §672)

Cela permet entre autres de remettre en perspective le moment bien connu où Wittgenstein soutient, dans le Tractatus, qu'il ne peut y avoir de propositions éthiques. Le but de la philosophie, pour lui, est la clarification logique des propositions. La philosophie elle-même est non pas un corps de doctrine, mais une activité: l'activité qui consiste à rendre claires nos pensées. De cette description de la tâche de la philosophie, il s'ensuit qu'il ne peut exister de philosophie morale que s'il existe un corpus de propositions qu'il reviendrait à la philosophie morale de clarifier. Pour Wittgenstein, il n'existe donc pas de partie de la philosophie qu'on puisse identifier comme "philosophie morale». Pourtant, bien que le Tractatus nie l'existence de propositions éthiques, Wittgenstein décrit ce livre comme étant pourvu d'une visée éthique - non pour signaler toutefois que ce livre contient des jugements moraux. Sa position est (alors et par la suite) qu'un ouvrage, par exemple un roman, peut avoir un objectif moral en dépit de l'absence de tout contenu ou théorisation moraux. Tel est l'effet du Tractatus, sa valeur (Wert) et son importance: nous aider à voir le monde correctement.

Ce thème de la perception morale, tout comme la critique de l'idée même d'une philosophie morale, se développent dans sa seconde philosophie.

Wittgenstein s'intéresse, par exemple dans ses cours de Cambridge des années 1930, à ce qui relie entre elles les significations de «bon» dans leurs différents contextes. Il existe des transitions graduelles d'une 
signification à une autre; des choses dites «bonnes» dans des contextes différents peuvent être liées entre elles par une série dont chaque membre entretient une similarité avec des membres contigus dans la série, sans forcément ressembler à des membres plus éloignés dans la série: il n'y a pas quelque chose de commun à toutes les choses bonnes. Cette réflexion sur le mot «bon» est une application de la critique générale de l'idée qu'il y aurait quelque chose de commun à tous les cas auxquels nous appliquons un terme général (bon). Wittgenstein, par sa notion de ressemblance de famille, récuse également l'idée de concepts éthiques et d'une philosophie morale. Il s'agit d' "exploration» éthique - non d'une réalité éthique à découvrir -, c'est-à-dire: de la façon dont nos préoccupations éthiques sont inscrites dans notre langage et notre vie, dans un ensemble de mots plus étendu que le vocabulaire éthique, et de ses rapports complexes avec une variété d'institutions et de pratiques qu'il reste à percevoir correctement. Pour décrire la compréhension éthique, il faudrait décrire tout cela, tous ces usages de mots particuliers, dont une définition générale ne peut rendre compte, et, pour décrire notre pratique langagière, tout l'arrière-plan de la forme de vie.

Il s'agit alors non pas tant de jugement moral et de compréhension que de perception et de vision synoptique: une perception ordinaire, par un ersatz philosophique «objectivant». Wittgenstein suggère dans sa seconde philosophie une approche gestaltiste en morale, par la nécessité d'un ressorti de la situation sur un arrière-plan narratif. Diamond définit cette approche ainsi:

Nos conceptions morales particulières émergent sur un arrièreplan plus général de pensée et de sensibilité. Nous différons dans notre façon de laisser (ou pas) les concepts moraux agencer notre vie et nos relations aux autres, dans la façon dont ces concepts structurent nos récits de ce que nous avons fait ou vécu.

(2006: 83-84)

Les éléments du vocabulaire éthique n'ont de sens que dans le contexte de nos usages et d'une forme de vie, ou, plutôt, prennent vie sur l'arrière-plan (celui de la praxis) qui «donne aux mots leur sens» - un sens moral qui n'est jamais fixé, et toujours dépendant de «nos" pratiques. "Ce n'est que dans la pratique du langage qu'un mot peut avoir une signification" (Wittgenstein, 1997: 344).

Le sens non seulement est défini par l'usage, ou le contexte (comme l'ont reconnu nombre d'analyses du langage), mais il est également inscrit et perceptible uniquement sur l'arrière-plan de la pratique du langage, qui se modifie par ce que nous en faisons.

"Beau» est lié à un jeu particulier. De même en éthique: la signification du mot «bon» est liée avec l'acte même qu'il modifie. Nous ne pouvons établir la signification du mot "beauté" qu'en considérant comment nous en faisons usage.

(Wittgenstein, 1979: 35)

On pourrait alors être tenté de tirer l'éthique vers une ontologie particulariste - qui mettrait des particuliers abstraits (tirés par exemple de la perception) au centre d'une théorie des valeurs ou d'un réalisme des particuliers. Mais ce serait encore perdre le sens de l'idée de ressemblance de famille, qui est précisément la négation d'une ontologie. Wittgenstein critique la pulsion de généralité:

La tendance à chercher quelque chose de commun à toutes les entités que nous subsumons communément sous un terme général. L'idée qu'un concept général est une propriété commune à ses cas particuliers se rattache à d'autres idées primitives et trop simples sur la structure du langage. (1996: 57-58)

Iris Murdoch, dans "Vision and Choice in Morality", évoque à ce propos l'importance de l'attention en morale (care: faire attention, être attentionné 3 ). Murdoch présente ainsi les différences en morale en termes de différences de Gestalt. Elle critique l'idée classique de la perception d'un objet par un concept:

Les différences morales ressemblent moins ici à des différences de choix, et plus à des différences de vision. En d'autres termes, un concept moral ressemble moins à un anneau mobile et extensible posé sur un certain domaine de faits, et plus à une différence de Gestalt. Nous différons, non seulement parce que nous sélectionnons différents objets à partir du même monde, mais parce que nous voyons des mondes différents. (1997) 
Il n'y a pas de concepts moraux univoques qu'il ne resterait qu'à appliquer à la réalité pour délimiter des objets; bien plutôt, nos concepts dépendent, dans leur application même, de la vision du «domaine», de la narration ou de la description que nous en donnons, de notre intérêt personnel et de notre désir d'exploration (de ce qui est important pour nous). Dans l'idée d'importance, nous avons une autre formulation du souci éthique: ce qui est important (matter) pour nous, ce qui compte.

\section{LA COMPÉTENCE ÉTHIQUE COMME CAPACITÉ PERCEPTIVE}

Ce rapport de la définition de l'éthique à ce qui compte a été mis en évidence par Harry Frankfurt, dans The Importance of What We Care About (1988) et par Stanley Cavell, dans un tout autre domaine, à propos du cinéma et des films qui comptent pour nous:

La morale que je tire est la suivante: pour répondre à la question «qu'advient-il des objets quand ils sont filmés et projetés?" - de même qu'à celle-ci: "qu'advient-il à des personnes données, à des lieux précis, à des sujets et à des motifs, quand ils sont filmés par tel ou tel cinéaste?» - il n'existe qu'une seule source de données, c'est-à-dire l'apparition et la signification de ces objets, de ces personnes, que l'on trouvera en fait dans la suite de films, ou de passages de films, qui comptent pour nous. (Cavell, 2003: 79)

L'attention que prône Wittgenstein est aussi cette attention spécifique à l'importance non visible des choses et des moments, à la dissimulation de l'importance dans notre vie ordinaire. Redéfinir la morale à partir de l'importance et de son lien à la vulnérabilité de notre expérience pourrait aider alors à constituer, en un sens nouveau, l'éthique du particulier. On peut renvoyer à une série ("cluster») de termes, ce jeu de langage de l'importance et de l'attention, de ce qui compte. Notre capacité d'attention devient pour Murdoch «une version détachée, non sentimentale, non égoïste, objective de l'attention» (1997). Elle est le résultat du développement d'une capacité perceptive: voir le détachement du détail, du geste, sur un arrière-plan.
La philosophie morale doit alors modifier son champ d'études, de l'examen de concepts généraux à l'examen des visions particulières, des «configurations» de pensée des individus

[...] qui se manifestent continûment dans leurs réactions et leurs conversations. Ces choses, qui peuvent être montrées ouvertement et de façon intelligible ou élaborées intimement et devinées, constituent ce qu'on peut appeler la texture d'être d'un homme, ou la nature de sa vision personnelle. (ibid.)

C'est bien dans l'usage du langage ("choix» des expressions, style de conversation) que se montre ou s'élabore la vision morale d'une personne, qui, pour Murdoch, n'est pas tant un point de vue théorique qu'une texture d'être (la texture pouvant être visuelle, sonore et tactile). Cette texture a à voir non pas avec les choix moraux, mais avec «ce qui importe» et qui fait et exprime les différences entre individus.

Nous ne pouvons pas voir l'intérêt moral de la littérature à moins de reconnaître les gestes, les manières, les habitudes, les tours de langage, les tours de pensée, les styles de visage, comme moralement expressifs - d'un individu ou d'un peuple. La description intelligente de ces choses fait partie de la description intelligente, aiguisée, de la vie, de ce qui importe, de ce qui fait la différence, dans les vies humaines. (Diamond, 2004: 507)

Ce sont ces différences qui doivent être l'objet de "la description intelligente, aiguisée, de la vie». Cette vie humaine renvoie à la forme de vie wittgensteinienne, qui définit aussi une texture. Notons également la texture ouverte définie à la même époque par F. Waismann (1945), à propos de la sensibilité de nos mots et de nos énoncés à leurs usages. La texture désigne alors une réalité instable, qui peut être fixée non par des concepts ou par des objets déterminés, mais par la reconnaissance de gestes, de manières, de styles. La forme de vie s'avère, prise du point de vue de l'éthique, définie par la perception - l'attention à des textures ou à des motifs moraux (ceux de Henry James, décrits par Diamond et Nussbaum dans leurs essais consacrés à cet auteur). Ces motifs sont perçus comme «moralement expressifs». Ce qui est perçu, ce sont donc non pas des 
objets ou une réalité morale (des valeurs), mais une expression morale, qui n'est possible et lisible que sur l'arrière-plan de la forme de vie. La littérature est le lieu privilégié de la perception morale, par la création d'un arrière-plan qui la rend possible, qui fait apparaître les différences importantes (signifiantes).

La définition de la compétence éthique en termes de perception affinée et agissante (contre la capacité à juger, argumenter et choisir) est reprise par Martha Nussbaum ${ }^{4}$. Pour elle, la morale est bien affaire de perception et d'attention, et non d'argument. Une objection que l'on pourra faire à son approche est qu'alors on revient à une opposition caricaturale entre le sentiment et la raison. Mais ce qui compte ici est plutôt le recentrement de la question éthique sur une forme de psychologie morale, fondée sur une perception fine et intelligemment éduquée, qui associe intimement - comme Jane Austen - sens et sensibilité.

L'éducation produit les significations. On peut penser, par exemple, à la vie de Hobart Wilson telle qu'elle est racontée dans l'article du Washington Post cité par Diamond dans "Différences et distances morales» (2006), ou aux caractères tels qu'ils sont décrits chez Henry James, qui nous apprend à les voir correctement. Dans sa préface à Ce que savait Maisie, James note: «l'effort pour voir réellement et peindre réellement n'est pas une mince affaire face à la force constante qui travaille à tout embrouiller" (2004: 165166). Ce roman est entièrement une critique de la perception, par la description d' «un monde social où la perception de la vie est caractérisée par l'incapacité à voir ou à jauger la vivacité d'esprit de Maisie» (ibid.). C'est pourquoi l'idée de description ou de vision ne suffit plus pour rendre compte de la vision morale: elle consiste à voir non pas des objets ou même des situations, mais les possibilités et significations qui émergent dans les choses; elle consiste à anticiper, à improviser (dit Diamond) à chaque instant dans la perception. La perception est alors active, non au sens kantien où elle serait conceptualisée, mais parce qu'elle est constamment changement de perspective, improvisation morale sur les gestes d'autrui, invitation à l'expression.
Voir les possibilités dans les choses est l'affaire d'une transformation dans la perception qu'on en a. Les possibilités ne se donnent pour ainsi dire que sous une contrainte.

(Diamond, 2004: 426)

Diamond reprend à Wittgenstein l'idée que nous avons affaire non aux phénomènes, mais aux «possibilités des phénomènes» (2004: \$90). Notre capacité d'expression morale, comme l'a dit Charles Taylor, s'enracine dans une forme de vie plastique, vulnérable à nos bons et mauvais usages du langage. C'est la forme de vie (au sens naturel, et indissolublement social, défini par Cavell) qui détermine la structure éthique de l'expression, laquelle inversement la retravaille et lui donne forme. Le type d'intérêt, de soin, que nous avons des autres et l'importance que nous leur donnons n'existent que dans la possibilité du dévoilement (réussi ou raté) de soi - dans l'expression morale.

Pour reconnaître réciproquement notre disposition à communiquer, présupposée dans toutes nos activités expressives, nous devons être capables de nous "lire» les uns les autres. Nos désirs doivent être manifestes pour les autres. C'est le niveau naturel de l'expression, sur lequel repose l'expression véritable. La mimique et le style s'appuient là-dessus [...]. Mais il n'y aurait rien sur quoi s'appuyer si nos désirs n'étaient pas incarnés dans l'espace public, dans ce que nous faisons et essayons de faire, dans l'arrière-plan naturel du dévoilement de soi, que l'expression humaine travaille sans fin. (Taylor, 1997: 86)

Ce qui est décrit sur un mode sceptique chez Cavell (la difficulté et le refus de l'expression) l'est sur un mode herméneutique par Taylor, mais l'un et l'autre aboutissent à un questionnement moral sur l'expression, la constitution du style, l'éducation de soi et des autres par l'apprentissage de l'attention aux expressions d'autrui: «Les expressions humaines, la silhouette humaine doivent, pour être saisies, être lues" (Cavell, 1996: 508). Cette lecture de l'expression, qui permet de répondre, est un produit de l'attention et du «care». Elle est le résultat d'une éducation de la sensibilité. Au thème perceptuel et trop statique de l'arrière-plan, on pourra préférer ceux de la texture, 
du motif et du grouillement vital, ou, comme dans les Fiches, celui de la place et des connexions ( La douleur occupe telle place dans notre vie, elle a telles connexions» [1967: §533]).

L'arrière-plan de la forme de vie n'est ni causal ni figé comme un décor, mais vivant, mobile. On peut encore une fois en appeler aux formes de vie (life forms, dit Cavell, au lieu de forms of life): les formes que prend notre vie sous le regard attentif. Le "tourbillon" de la vie dans le langage et non pas, par exemple, un corps de significations ou de règles sociales. Ici s'opposeraient deux représentations éthiques et deux approches de la perception morale: celle de l'arrièreplan (voir Searle [1995], pour qui les institutions constituent l'arrière-plan fixe qui nous permet d'interpréter le langage, de percevoir et de suivre des règles sociales) et celle de la texture perceptive de la vie. Le terme d'arrière-plan (Hintergrund) apparaît chez Wittgenstein pour désigner un arrière-plan de la description, qui fait ressortir la nature des actions, et non, comme le suggère Searle, pour expliquer quoi que ce soit. L'arrière-plan ne peut avoir de rôle causal, car il est le langage même dans son instabilité et sa sensibilité à la pratique:

Comment pourrait-on décrire la façon d'agir humaine?

Seulement en montrant comment les actions de la diversité des êtres humains se mêlent en un grouillement. Ce n'est pas ce qu'un individu fait, mais tout l'ensemble grouillant qui constitue l'arrière-plan sur lequel nous voyons l'action.

(Wittgenstein, 1967: §567)

Nous percevons l'action, mais prise au milieu d'un grouillement, du tourbillon de la forme de vie sur lequel elle ressort et devient sensible, importante. Ce n'est pas la même chose de dire que l'application de la règle est causalement déterminée par un arrière-plan, et de dire qu'elle est à décrire dans l'arrière-plan d'actions et de connexions humaines (Diamond, 1989; Laugier, 2006c). C'est toute la différence entre une conception gestaltiste et descriptive de l'éthique et une conception "conformiste» qui voudrait justifier nos actions par un accord préalable sur des règles ${ }^{5}$. L'arrière-plan ne donne ni ne détermine une signification éthique (car il n'y en a pas), mais permet d'avoir une perception de ce qui est important et signifiant pour nous (du moment important). Wittgenstein mentionne, dans les Remarques mêlées, "l'arrière-plan sur lequel ce que je peux exprimer reçoit une signification" (1984: 16). Le sens de l'action se donne par perception sur l'arrièreplan de la forme de vie. L'arrière-plan «accepté», donné, ne détermine pas nos actions (pas de causalité) mais nous permet de les voir clairement.

\section{LA PERCEPTION MORALE DU SENS}

\section{COMME AVENTURE}

Martha Nussbaum revendique ainsi en morale la recherche d'un "équilibre perceptif " parallèle à l'équilibre réfléchi de John Rawls. Elle attribue à James une "vision morale" qui, chez elle, devient un universel:

Le roman construit un modèle de style de raisonnement éthique

qui est lié au contexte sans être relativiste, et nous donne des

impératifs concrets susceptibles de devenir des universaux.

Toute situation particulière devrait être ramenée, dans la tradition des éthiques de la vertu, à une «idée générale» de la vie réussie. C'est le seul moyen de valider «un raisonnement propre au roman qui ne pourrait être effectué par la théorie»: «la philosophie morale exige l'expérience de la lecture aimante et attentive de romans pour son propre

accomplissement» (ibid.: 26-27).

Par cette lecture «attentive» et, dirait-on, "caring", nous percevons les situations morales autrement, activement. Nussbaum revendique l'acquisition d'un équilibre perceptif par la littérature, d'une vision peaufinée ("fine-tuned»). Diamond ajoute que l'attention aux autres que donne la littérature ne nous fournit pas de nouvelles certitudes ou l'équivalent littéraire de théories, elle nous met en face d'une incertitude, d'un déséquilibre perceptif, ou encore aux prises avec ceux-ci. Nussbaum note pourtant que «la délibération humaine est constamment une aventure de la personnalité, lancée parmi des hasards terrifiants et des mystères effrayants» (ibid.: 142). En se focalisant 
sur une conception étroite de l'éthique, ce qu'on risque, c'est de passer à côté de l'aventure, c'est-à-dire de manquer une dimension de la morale, par manque d'attention: plus précisément, le visage de la pensée morale, «ce à quoi ressemble la vie morale» (Diamond, 2004: 36).

Ici intervient l'aventure conceptuelle comme composante de la perception morale. Il y a de l'aventure dans toute situation qui mêle l'incertitude, l'instabilité et «le sens aiguisé de la vie». Henry James explicite cette forme aventureuse que prend la pensée morale:

Une "aventure" humaine, personnelle n'est pas une chose a priori, positive, absolue et inextensible, mais juste une question de relation et d'appréciation - dans les faits, c'est un nom que nous donnons, avec à propos, à tout passage, toute situation qui a ajouté le goût tranchant de l'incertitude à un sens aiguisé de la vie. C'est pourquoi la chose est, tout à fait admirablement, une question d'interprétation et de conditions particulières; et faute d'une perception de celles-ci, les aventures les plus prodigieuses peuvent vulgairement compter pour rien.

(1980: 307)

La perception du sens est activité, mobilité et improvisation. Or, c'est une mobilité sensible autant qu'intellectuelle. Wittgenstein parle à ce propos, dans ses manuscrits, d'«une mobilité spirituelle» (Nachlass, Ms 136, 61b). Mais il s'agit alors moins d'un équilibre, d'une juste vision (prônés par l'éthique des vertus) que d'un déséquilibre et d'une intensité qui poussent les frontières de l'éthique à la limite.

Le lecteur inattentif rate donc doublement: il manque l'aventure des personnages (pour lui, «ils comptent pour rien»), et il manque sa propre aventure comme lecteur.

(Diamond, 2004: 425)

Car c'est le côté négatif de l'attention qui nous fait le mieux comprendre le (non) sens de l'éthique. L'absence d'attention (de "care»), le manque de perception de l'importance font «manquer l'aventure». Ainsi, on peut voir la vie morale comme une aventure à la fois conceptuelle (on étend ses concepts) et sensible (on s'expose).
La littérature, selon Diamond, nous donne en effet l'occasion de pousser à la limite nos capacités de compréhension, de nous mettre à la place d'autrui. C'est sur ce point - la capacité à comprendre autrui, et par exemple celui qui dit le non-sens - que Diamond introduit un élément d'interprétation du Tractatus de Wittgenstein devenu célèbre, en reprenant le pénultième paragraphe.

Mes propositions sont élucidantes en cela que celui qui me comprend les reconnaît à la fin pour des non-sens, si, passant par elles - sur elles - par-dessus elles, il est monté pour en sortir. Il faut qu'il surmonte ces propositions; alors il acquiert une juste vision du monde. (1993: 6.54)

Diamond attire notre attention sur l'expression étrange: "celui qui me comprend». Il ne s'agit pas de comprendre le non-sens (ce qui est impossible, bien sûr), mais celui qui le dit, l'auteur du Tractatus. Mais qu'est-ce alors que «comprendre celui qui dit le nonsens»?

Vous devez lire ses propositions dénuées de sens et essayer de comprendre non pas elles mais leur auteur; c'est précisément ainsi qu'il estime devoir répondre aux non-sens des philosophes: en comprenant non leurs propositions mais eux-mêmes.

(Diamond, 2000)

La compréhension du non-sens éthique passe par la compréhension de celui qui dit ces propositions. Cela passe par une question autre, qui est celle de savoir ce qu'est comprendre quelqu'un (en général). Nous savons ce qu'est comprendre quelqu'un (et, dans la plupart des cas, ce n'est pas différent de comprendre ce qu'il dit). Mais ce n'est pas saisir un contenu de son esprit, ni accéder à l'intérieur. «Il n'y a, comme je l'ai dit, pas d'intérieur. Mais comprendre une personne qui prononce du non-sens revient à aller aussi loin que possible avec l'idée qu'il y en a un" (ibid.: 157). On retrouve le sens de l'aventure: il ne s'agit pas d'empathie ou de quelque chose de purement émotif, comme le dit Wittgenstein dans les «Leçons sur la croyance religieuse» (1971).

Imaginez quelqu'un qui, avant d'aller en Chine, risquant de ne plus jamais me revoir, me dise "Il se pourrait que nous nous 
voyions une fois morts" - dirais-je nécessairement que je ne le comprends pas? Je dirais peutêtre (j’en aurais le désir) «Oui, je le comprends tout à fait». Lewy: dans ce cas, vous pourriez penser simplement qu'il a exprimé une certaine attitude. Je dirais "Non, ce n'est pas la même chose que de dire "J'ai beaucoup d'affection pour vous", et il se peut que ce ne soit pas la même chose que de dire quoi que ce soit d'autre». Cela dit ce que cela dit.

Il s'agit, dans le rapport à la littérature, d'aller le plus loin possible de façon à donner sens au comportement et aux paroles d'autrui perçus comme incompréhensibles. Un usage de la littérature consisterait alors à "prendre la vie morale en tant que lieu d'aventure", et à faire de la lecture elle-même notre aventure. Une telle aventure requiert des qualités spécifiques: celles, dit Nussbaum, de l'improvisation morale. Elle s'oppose, note Diamond, à l'inattention morale, à l'étroitesse d'esprit et au refus de l'aventure. Ici, on peut faire intervenir le scepticisme. Ce qui est en cause - et qui est représenté ou «transposé» dans le scepticisme philosophique, le doute par exemple sur la possibilité de connaître autrui -, c'est notre difficulté essentielle à connaitre autrui et à nous laisser connaître par lui. C'est cette difficulté que Cavell décrit lorsqu'il évoque la tentation de l'inexpressivité et de l'isolement, notre incapacité à aller au-delà de nos réactions naturelles pour connaître l'autre, nous mettre à sa place, sortir des limites de notre entendement et du sens commun.

Notre capacité à communiquer avec lui dépend de sa "compréhension naturelle», de sa «réaction naturelle» à nos instructions et à nos gestes. Elle dépend donc de notre accord mutuel dans les jugements. Cet accord nous conduit remarquablement loin sur le chemin d'une compréhension mutuelle, mais il a ses limites; limites qui, pourrait-on dire, ne sont pas seulement celles de la connaissance, mais celles de l'expérience. Et lorsque ces limites sont atteintes, lorsque nos accords sont dissonants, je ne peux plus revenir en arrière, vers un terrain plus solide. (1996: 184)

Cavell s'intéresse à l'émergence de désaccords radicaux en morale. C'est cette possibilité de l'incompréhension radicale qui définit la perception éthique: la question morale passe par nos accords "dans le langage» (Laugier, 2006a), mais aussi par un radical de désaccord et d'incompréhension, de distance, de sentiment de non-sens (comme réaction naturelle, indignation ou révolte).

Non seulement lui ne me "reçoit» pas, parce que ses réactions naturelles ne sont pas les miennes; mais c'est mon propre entendement qui s'avère incapable de passer outre ce que mes propres réactions naturelles peuvent supporter. (Cavell, ibid.)

Diamond s'intéresse à notre capacité de reconnaitre quand les mots de quelqu'un exhibent une manière de quitter le monde conceptuel commun. Cette capacité est liée à notre capacité de perdre - et, symétriquement, d'étendre - nos concepts (moraux), de les utiliser dans de nouveaux contextes. Une telle extension, comme la mesure de ses limites, est le travail de l'imagination morale, de notre capacité/incapacité à nous mettre dans une situation et à comprendre les paroles d'autrui, qui apparaît au moment où nous mesurons notre distance avec la vision morale d'autrui ${ }^{6}$.

La question est non plus seulement, alors, l'imagination et la sensibilité comme sources de connaissance morale, mais aussi la mise en évidence sceptique - de la perte de nos concepts, la difficulté à les mettre en œuvre dans de nouveaux contextes, un autre monde conceptuel. Diamond a étudié ce point dans son essai «Losing your concepts».

Une sensibilité au monde conceptuel dans lequel se situent les remarques de quelqu'un est un moment de la sensibilité humaine aux mots. [...] Cavell lui-même s'intéressait ici au fait que nous partagions certaines choses; je m'intéresse maintenant aussi à notre capacité de reconnaître quand les mots de quelqu'un montrent, ou semblent montrer, une manière de quitter le monde conceptuel commun. [...] Vous reconnaître, vous et la personne à laquelle vous parlez, comme partageant le même monde moral ce n'est pas l'imaginer comme quelqu'un avec qui vous atteindrez l'accord sur des questions morales. Vous considérez que vous ne partagez pas le même monde moral si votre réaction à ce qu'il dit, par exemple, "Comment a-t-il pu invoquer cela ici? Comment peut-il aller jusqu'à penser cela 
comme pertinent [...]. Quelle vie vit-il, quelle vie dans laquelle une telle discussion peut se poursuivre?»

(1988: 273; trad. de E. Halais)

Pour Diamond, cette capacité est spécifique à la réflexion éthique, aux expériences de pensée propres à l'éthique (se mettre à la place de quelqu'un par exemple). Elle concerne l'ensemble de la pensée: la capacité à projeter nos mots et concepts dans des contextes nouveaux, à être toujours prêts à perdre nos concepts, à voir de nouvelles différences et distances. C'est une sensibilité «à des formes de la vie conceptuelle». Il n'y a pas, comme le fait parfois Nussbaum, à séparer la vie intellectuelle et le sentiment. C'est plutôt le caractère sensible des concepts moraux et le caractère improvisé de l'activité conceptuelle qui sont mis en évidence.

Dans un de ses récents textes, "The Difficulty of Reality and the Difficulty of Philosophy» (2003), Diamond évoque ainsi un aspect de la vie éthique qui n'a de place et d'expression que dans la littérature: le fait que certains aspects de la réalité soient insupportables, ne puissent même pas être pensés sauf avec grande difficulté pour la philosophie. Le scepticisme est alors l'expression de notre refus - et pas seulement de notre incapacité - de penser certaines choses, de nous mettre à la place d'un autre qui dirait du non-sens. Diamond utilise pour mettre cela en évidence un essai de J.M. Coetzee, «La vie des animaux» (texte inclus dans son roman Elizabeth Costello), qui lui-même fait intervenir un réseau de textes - Kafka et son Rapport pour une Académie, Köhler et son compte rendu d'expérience sur les singes, Nagel et sa chauve-souris, Descartes et son cogito - autour d'une écrivaine australienne, Elizabeth Costello, venue donner une conférence aux États-Unis sur les droits des animaux. Coetzee et Diamond explorent notre capacité à comprendre l'autre et montrent que le texte de Kafka - qui donne la parole à un singe, Peter le Rouge, présenté à une académie scientifique à laquelle il s'adresse, lui fournissant «un rapport sur son passé simien» - permet précisément de se mettre à la place d'un autre radicalement étranger. Elizabeth Costello soutient, dans sa conférence devant le public américain (se plaçant ellemême, de façon plutôt ridicule et inconfortable, dans la position du singe faisant son «rapport à l'académie»), que l'expérience que nous donne la littérature est celle de la sympathie, la possibilité d'imaginer ce qu'est réellement être à la place d'un être étranger. Elle se livre à un parallèle, qui paraît alors scandaleux et incompréhensible, entre la «vie des animaux» et la Shoah. Comprendre cette étrange comparaison (ne pas être choqué par elle, ni désireux de prendre une distance avec elle), c'est se rendre capable de vivre l'aventure et la souffrance de cette femme et de comprendre quel est exactement le point que veut mettre en évidence Coetzee. Nous refusons de penser certains éléments de la réalité, de nous imaginer à la place d'un autre, non par incapacité ou défaut conceptuel, mais par refus de comprendre l'autre qui dit le non-sens ${ }^{7}$. Pourtant, note Costello, nous sommes capables pour un moment d'envisager quelque chose d'aussi inimaginable que notre propre mort, d'être saisi par cela. Pourquoi ne pas imaginer qu'on est Peter le Rouge, un singe? Coetzee ne parle pas par l'intermédiaire de Costello, mais il nous permet d' "entrer dans son esprit», de la comprendre, si étrange et loin qu'elle soit.

La question que pose Elizabeth Costello est bien celle - radicalisée - de la possibilité d'étendre nos concepts, jusqu'à la compréhension du non-sens.

Il y a des gens qui sont capables de s'imaginer comme quelqu'un d'autre, il y en a d'autres qui n'ont pas cette capacité, enfin il y a des gens qui ont cette capacité mais qui font le choix de ne pas l'exercer. Malgré Thomas Nagel, qui est sans doute un brave homme, malgré Thomas d'Aquin et Descartes, avec qui j'ai plus de mal à sympathiser, il n'y a pas de limite à notre capacité à nous projeter à l'intérieur de l'être d'un autre. Il n'est pas de bornes à l'imagination sympathique. (Coetzee, 2004)

C'est ce caractère aventureux de la perception morale qui lui permet de "perdre [ses] concepts" et de les étendre par la même occasion. On comprend ainsi la nature de l'expérience elle-même, qui se révèle définie par notre capacité d'attention. Cette attention 
est une capacité à voir le détail, le geste expressif, même si ce n'est pas forcément une vision claire, ni exhaustive - une attention à l'importance (what matters), à ce qui compte dans les expressions et les styles d'autrui, à ce qui fait et exprime les différences entre personnes -, et le rapport de chacun à son expérience qu'il faut alors décrire. L'attention à l'ordinaire est alors la perception des textures ou des motifs moraux (ceux de Henry James par exemple, comme le motif «international»). La littérature est le lieu privilégié de cette perception des expressions, par la création d'un arrière-plan qui fasse apparaittre les différences importantes entre les expériences et les expressions qui sont présentées et de nouvelles configurations et textures morales.

Il s'agit, pour conclure, d'une compétence qui est affaire non seulement de connaissance ou de raisonnement, mais aussi d'apprentissage de l'expression adéquate et particulière et d'éducation de la sensibilité; éducation de la sensibilité du lecteur par l'auteur, qui lui rend perceptible telle ou telle situation, tel caractère, en le plaçant (le décrivant) dans le cadre adéquat. Le roman nous apprend alors à regarder la vie comme «la scène de l'aventure et de l'improvisation». L'apprentissage que nous offre le roman est une initiation aux modes d'expression adéquats, linguistiques ou autres, à une forme de vie: une formation sensible par l'exemplarité. Le roman forme alors notre capacité à (lire) l'expression morale. Cette capacité d'expression est conceptuelle et langagière - capacité à faire bon usage des mots pour décrire l'expérience. Il s'agit alors de l'expression de l'expérience: quand et comment faire confiance à son expérience, découvrir sa validité propre.

L'attention aux expressions que propose et suscite la littérature de James ne nous donne pas de certitudes, mais fait émerger l'incertitude: elle fait de l'expérience même de la lecture une aventure au sens strict (ou, pour parler comme Emerson, qui a inspiré Henry James et son frère William, «true romance»). Il y a de l'aventure, selon James, dans toute situation qui mêle l'incertitude et le "goût de la vie»: la lecture est de l'ordre de l'aventure au sens propre, non métaphorique. L'expérience elle-même, si on lui fait confiance, devient alors une aventure. Refuser cette confiance, la vie, c'est passer à côté de l'aventure celle des personnages, et la sienne propre. L'absence d'attention à l'expérience, le manque de perception de l'importance font passer à côté de l'aventure.

Ainsi, on peut voir l'expérience comme une aventure à la fois conceptuelle et sensible - dit autrement: comme à la fois passive (on se laisse transformer, toucher) et agentive (la mobilité spirituelle et la perception agissante). Il n'y a plus à séparer vie conceptuelle et affection, comme il n'y a pas à séparer, dans l'expérience morale, la pensée (la spontanéité) et la réceptivité (la vulnérabilité au réel et aux autres, au risque du déséquilibre perceptif et de l'incompréhension). C'est cela, dit James, qui constitue l'expérience.

Le pouvoir de deviner ce qu'on n'a pas vu à partir du visible, de suivre les implications des choses, de juger l'ensemble par son motif, l'état où vous ressentez si complètement la vie en général que vous êtes en bonne voie pour en connaître les moindres recoins - on peut presque dire que cet agrégat (cluster) de dons constitue l'expérience. (1884)

James ajoute qu'il faut alors que rien n'échappe, ne se perde. C'est par cette volonté d'appropriation et de re-collection de sa propre expérience que l'on peut définir, en fin de compte, la perception morale. 


\section{N O TES}

1. Sauf indication contraire, nous avons traduit les citations tirées des textes anglais.

2. Dans un «Entretien avec J. Bouveresse», 2000 (texte inédit; trad. de M. Coelho).

3. À ce propos, voir Paperman et Laugier (2005).

4. Dans plusieurs essais de Nussbaum (1990): "The Discernment of Perception ", "Finely Aware and Richly Responsible”: Moral Attention and the Moral Task of Literature Perception", et "La littérature comme philosophie morale. La fêlure dans le cristal: La Coupe d'or de James".

5. Il y a ici un parallèle entre la procédure rawlsienne (l'accord dans la position originelle et l'équilibre réfléchi) et l'équilibre perceptif. 6. On peut en avoir une idée en lisant les témoignages qu'elle cite, dans son essai "Différences et distances morales» (2006), de lecteurs du Washington Post après la publication de l'article de Chip Brown sur la vie de Hobart Wilson.

7. On pourrait aussi bien prendre comme exemple, ici, Disgrâce (1999) du même auteur.

\section{RÉFÉREN CES BIBLIO G RAPH IQ U ES}

CAVELL, S. [1991]: Une nouvelle Amérique encore inapprochable, trad. de S. Laugier, Combas, Éd. de l'éclat;

[1996]: Les Voix de la raison, Paris, Seuil;

[2003] : "Qu'advient-il des choses à l'écran?» Le cinéma nous rendil meilleurs?, trad. de C. Fournier et E. Domenach, Paris, Bayard, 61-80. CoetzeE, J. M. [(2003) 2004] : Elizabeth Costello, "La vie des animaux", trad. de C. Lauga du Plessis, Paris, Seuil.

DiAmOND, C. [1988] : «Losing your Concepts », Ethics, vol.98, n², 255-277;

[1989]: "Rules: looking in the right place», dans D.Z. Phillips et P. Winch (dir.), Attention to Particulars, New York, St Martin's Press; [(1991) 2004]: Wittgenstein. L'Esprit réaliste, trad. de E. Halais et

J.Y. Mondon, Paris, PUF; [(1997) 2006]: «Différences et distances morales", trad. de J. Y. Mondon et S. Laugier, dans S. Laugier (dir.), 53-94;

[2000]: "Ethics, imagination and the method of the Tractatus", dans A. Crary et R. Read (dir.), The New Wittgenstein, Londres, Routledge.

James, H. [1884]: The Art of Fiction. En ligne: http://en.wikiquote.org/ wiki/Henry_James (page consultée le 23 mai 2008); [[1909) 1981]: Les Ambassadeurs, vol. 1, trad. de G. Belmont,

Paris, Robert Laffont, coll. "Bouquins"; [(1934) 1980]: La Création littéraire, trad. de M. F. Cachin, Paris, Denoël-Gauthier;

[2004]: Ce que savait Maisie, Paris, Éd. 10-18.

LAUGIER, S. [2006a] : «Wittgenstein and Cavell: Anthropology,
Skepticism, and Politics", dans A. Norris (dir.), The Claim to Community: Essays on Stanley Cavell and Political Philosophy, Stanford (CA), Stanford University Press, 19-38;

[2006b]: "Concepts moraux, connaissance morale", dans S. Laugier (dir.), 147-195;

[2006c] : "Où se trouvent les règles?", dans C. Chauviré et S. Laugier (dir.), Lire Wittgenstein. Les Recherches Philosophiques, Paris, Vrin, 131-156.

LAUGIER, S. (dir.) [2006]: Éthique, littérature, vie humaine, Paris, PUF. Murdoch, I. [1997]: "Vision and Choice in Morality", Existentialists and Mystics: Writings on Philosophy and Literature, textes rassemblés par P. J. Conradi, Londres, Chatto and Windus, 76-98.

Nussbaum, M. [1990]: Love's Knowledge, Essays on Philosophy and Literature, Oxford, Oxford University Press;

[2006]: «La littérature comme philosophie morale. La fêlure

dans le cristal: La Coupe d'or de James", trad. de E. Halais et J. Y.

Mondon, dans S. Laugier (dir.), 19-51.

PAPERMAN, P. et S. LAUGIER (dir.) [2005]: Le Souci des autres. Éthique et politique $d u$ care, Paris, Éd. de L'EHESS.

PutnAm, H. [1994]: Words and Life, éd. et préface de J. Conant,

Cambridge (Mass.), Harvard University Press;

[2004] : Ethics without Ontology, Cambridge (Mass.), Harvard

University Press.

SEARLE, J. R. [1995]: The Construction of Social Reality, New York, Free

Press.

TAYLOR, C. [1997] : La Liberté des modernes, trad. de P. de Lara, Paris,

PUF.

WAISMANN, F. [(1945) 2004]: «La vérifiabilité», dans S. Laugier et

P. Wagner (dir.), Philosophie des sciences, tome I, Paris, Vrin.

WitTGenstein, L. [(1922) 1993]: Tractatus logico-philosophicus, trad. de

G.-G. Granger, Paris, Gallimard;

[(1953) 2004]: Recherches philosophiques, trad. de F. Dastur et alii,

Paris, Gallimard;

[(1954) 1997]: Cours de Cambridge, 1930-1932. Philosophica, tome

1, trad. de J.-P. Cometti, Mauvezin, TER;

[(1958) 1996]: Le Cahier bleu et le cahier brun, trad. de

M. Goldberg et J. Sackur, Paris, Gallimard;

[1967] : Fiches, trad. de J. Fauve, Paris, Gallimard;

- [1971]: "Leçons sur la croyance religieuse ", Leçons et

Conversations, trad. de J. Faure, Paris, Gallimard; [1979]: Cours de Cambridge, 1932-1935, notes de A. Ambrose,

Oxford, Oxford University Press; [(1980a) 1994]: Remarques sur la philosophie de la psychologie, trad. de G. Granel, Mauvezin, TER; [(1980b) 1984]: Remarques mêlées, trad. de G. Granel, Mauvezin, 